\title{
Discriminated avoidance vs classical conditioning: A two-phase model analysis*
}

\author{
WILLIAM F. PROKASY \\ University of Utah, Salt Lake City, Utah 84112
}

\begin{abstract}
The acquisition of discriminated avoidance and classically conditioned leg flexions in dogs and of discriminated avoidance leg flexion in cats was examined via a two-phase model of conditioning. Classically conditioned Ss uniformly required two operators during Phase 2, with the limit of the CR operator being greater than that of the $\overline{\mathrm{CR}}$ operator. Not all avoidance-trained Ss required two operators during Phase 2, but those who did also had a higher CR than $\overline{\mathrm{CR}}$ operator limit. The CR operator limit was higher for avoidance than for classical training, while the change rate was greater for classical training. The avoidance behavior of cats paralleled that of the dogs. It was concluded that the data generally conformed to views of Hilgard and Marquis and, more recently, of Bolles, but were contrary to what would be expected from two-factor theory.
\end{abstract}

The purpose of this paper is to employ the two-phase model (Prokasy, 1972, 1973; Prokasy et al, 1974) to compare and contrast discriminated avoidance with classically conditioned behavior and to determine the extent to which selected theories of avoidance behavior can account for the resulting pattern of parameter values. The dependent variable is the likelihood of leg flexion responses in dogs and cats.

As Wahlsten and Cole (1972) have noted, there are several accounts of the emergence and maintenance of discriminated avoidance behavior. Though seldom examined quantitatively, each of these accounts has implications for trial-by-trial changes in response probability for classical as well as discriminated avoidance conditioning.

One view (Bolles et al, 1966; Hilgard \& Marquis, $1940, \mathrm{pp} .58-62)$ is that two mechanisms are required to account for acquisition. The first, a consequence strictly of CS and UCS pairings, is necessary to account for initial CR emergence. The second is necessary to account for response maintenance. The observable operations correlated with these mechanisms are, respectively, CS- $\overline{\mathrm{CR}}-\mathrm{UCS}$ and CS-CR- $\overline{\mathrm{UCS}}$. Thus, early in training the CS-CR-UCS trial outcome will yield an increment in response probability. Later in training, increments are associated with the occurrence of the avoidance response. The role of CS- $\overline{C R}-\mathrm{UCS}$ in either incrementing or decrementing subsequent response likelihood in later stages of training is not clear. With the classical preparation, CS-CR-UCS should result in an increment in response probability early in training, as in the avoidance case. However, since the $C R$ is punished via the CS-CR-UCS trial outcome characteristic of classical conditioning, the mechanism for response maintenance is, if not removed, at least less efficient. As a result, one

\footnotetext{
*Supported by NSF Grant GN-12688 and a grant from the University Research Committee of the University of Utah.
}

would expect lower overall performance levels with the classical rather than the avoidance preparation.

Two-factor theory yields somewhat different predictions than does the preceding account. Paired trials, classical or avoidance, in the absence of the CR should result in increments in response probability. This is because the UCS elicits a reflex response in the presence of a fear-evoking or painful stimulus. The occurrence of the elicited response is correlated with the removal of the UCS and, consequently, with pain and/or fear reduction. Fear reduction is the reinforcement for a response, whether escape or avoidance. However, an avoidance response, in eliminating the UCS, also produces a decrement in fear which, in tum, results in a decrement in response probability for the next trial. Thus, while there might be an increment in response probability following CS-CR-UCS trials early in training (i.e., while fear is being acquired to the $\mathrm{CS}$ ), during later training that kind of trial should result in a reduction in response likelihood. In the classical preparation the effect of a CR is not easily specified. The $\mathrm{CR}$, by virtue of a following UCS, is punished, but the UCR, the same response, remains correlated with UCS removal. Depending upon response topography, there could be either increments or decrements. Whether or not the classical preparation should, considering this possible complication, result in lower levels of performance than should the avoidance preparation cannot, therefore, be specified.

Sheffield's contiguity theory (Sheffield, 1948), in spite of differences in assumed mechanisms, yields predictions similar to those of the two-factor theory. In both classical and avoidance training, response probability should increase following a CS-CR-UCS trial, but it should decrease following a CS-CR- $\overline{\mathrm{UCS}}$ trial. One contrast with the two-factor theory is that on 
CS-CR-UCS trials there should continue to be increments in response probability as a result of the fact that the CR and the UCR are not incompatible with each other. The Sheffield theory, then, does permit the prediction that classical training will result in higher performance than will avoidance training.

Bush and Mosteller (1955, pp. 237-258) and Theios (1971) have analyzed avoidance behavior with a two-operator linear model. It was assumed that response probability increased following both $\mathrm{CR}$ and $\overline{\mathrm{CR}}$ trials. Allowance was made for the possibility that the growth rates differed between $C R$ and $\bar{C} \bar{R}$ trials, but a common limit was assumed. Since it was employed strictly as a formal model, no implications exist for differentiation between the classical and avoidance preparations, although the method of parameter estimation employed by these investigators would permit differences in growth rate to emerge. Developed as a variant of Hull-Spence theory, Hoffman's model (Hoffman, 1965) yields predictions essentially the same as those of the Bush-Mosteller model, i.e., increments on both CR and $\overline{\mathrm{CR}}$ trials regardless of preparation.

The two-phase model, a variant of one first proposed by Norman (1963), is two-phase in that during Phase 1 no performance changes occur (i.e., response probability remains constant at a low initial value) and during Phase 2 increments and decrements can occur depending upon trial outcome. $P_{i}$, response probability on Trial $i$, is expressed as:

$$
\begin{array}{r}
P_{i}=P_{o}, i=1, K \\
P_{i}=P_{i-1}+\theta_{1}\left(\lambda_{1}-P_{i-1}\right), i=K+1, N, \\
\text { given CR on Trial } i-1 \\
P_{i}=P_{i-1}+\theta_{2}\left(\lambda_{2}-P_{i-1}\right), i=K+1, N, \\
\text { given } \overline{C R} \text { on Trial } i-1
\end{array}
$$

where $K$ is the trial on which $S$ switches from Phase 1 to Phase $2, \mathrm{P}_{\mathrm{o}}$ is the base level of response probability prior to conditioning, $\theta_{1}$ and $\theta_{2}$ are growth parameters, and $\lambda_{1}$ and $\lambda_{2}$ are limits. It will be noted that during Phase 2 one operator applies to $\mathrm{CR}$ trials and the other to $\overline{\mathrm{CR}}$ trials.

Two sets of data were employed in this study. The first set came from research reported by Wahlsten and Cole (1972). ${ }^{1}$ These investigators studied the acquisition of a leg flexion response in dogs with both classical and discriminated avoidance operations. The second set of data came from Nielson and Davis (1966), ${ }^{1}$ who studied the acquisition of leg flexion responses in cats in a discriminated avoidance situation.

\section{METHOD}

Experimental details of the research with dogs are provided by Wahlsten and Cole (1972, pp. 34-37). Briefly, two groups of
$\operatorname{dogs}(N=14$ in each group) were given a $1000-\mathrm{Hz}$ tone as a $\mathrm{CS}$ and a .5-sec electric shock to the lower right foreleg as a UCS. A CR was defined as a minimum leg flexion of $56 \mathrm{~mm}$ occurring within the 2-sec interstimulus interval. Ss in Group C received 50 trials per day of classical conditioning training, with the total number of trials per $S$ varying from 300 to 1200 . Group A received 50 trials per day of classical conditioning training, with the total number of trials per $S$ varying from 500 to 1250 . Training was terminated when, in the judgment of the $E$, response latency had stabilized over four sessions. The mean numbers of training trials (785.7 and 736.4 for Groups A and C, respectively) did not differ greatly.

Experimental details of the research with cats are provided by Nielson and Davis (1966). Briefly, 16 cats were given avoidance training at 25 trials a day until a criterion of $15 \mathrm{CRs}$ in a daily block of 25 trials was achieved. The total number of trials varied across Ss from 325 to 1525 . The CSs (2-sec duration) were either a $1000-\mathrm{Hz}$ tone or electrical stimulation of a substructure of the reticular formation (locus of CS had no noticeable effect on performance). The UCS, a 2 -sec train of pulses to S's right foreleg, was delivered $2 \mathrm{sec}$ after CS onset on all $\overline{\mathrm{C}} \overline{\mathrm{R}}$ trials. A CR was defined as any right foreleg movement during the 2-sec interstimulus interval which broke contact with a copper grid beneath the paw.

\section{RESULTS}

It was assumed arbitrarily that $P_{0}=0.0$. With that exception, the remaining parameter estimates were obtained with the use of the computer subroutine STEPIT (Chandler, 1969). Procedural details on the estimation procedure are provided by Prokasy (1973).

Best-fit parameters were obtained separately for each $S$ under four different assumptions:

$$
\begin{aligned}
& \text { A. } \theta_{1}=\theta_{2} ; \lambda_{1}=\lambda_{2} \\
& \text { B. } \theta_{1} \neq \theta_{2} ; \lambda_{1}=\lambda_{2} \\
& \text { C. } \theta_{1}=\theta_{2} ; \lambda_{1} \neq \lambda_{2} \\
& \text { D. } \theta_{1} \neq \theta_{2} ; \lambda_{1} \neq \lambda_{2}
\end{aligned}
$$

It will be noted that the model implied by Assumptions $B$ and $C$ are submodels of that implied by Assumption D. Similarly, the model implied by Assumption A is a submodel of those implied by Assumptions B and C. Under these circumstances, it is possible to employ a maximum likelihood ratio test to determine which assumption is sufficient to account for the data (Theios, 1968). Thus, for a given $S$ the likelihood of the protocol was obtained separately under Assumptions $\mathbf{A}$ and $\mathbf{B}$, and a chi-square was calculated as

$$
\chi^{2}=-2 \log _{e} \frac{L_{A}}{L_{B}}
$$

Since there is a difference of one parameter between tl : two assumptions, the chi-square has $1 \mathrm{df}$. Th assumption that required the greater number of parameters was assumed to apply to a protocol if the obtained chi-square (with $1 \mathrm{df}$ ) exceeded 3.8. The 
method is described more fully elsewhere (Prokasy, 1973; Theios, 1968).

The results for the dogs, Groups $\mathrm{A}$ and $\mathrm{C}$, are provided in Table 1, while those for the cats are presented in Table $2 .^{2}$ The assumption that was found to be sufficient for each $\mathrm{S}$ is also indicated.

All Group C Ss required two operators. Of these, 11 had two different $\lambda$ s and a common $\theta$, while the remaining 3 required different $\theta$ s and different $\lambda$ s. Without exception $\lambda_{1}>\lambda_{2}$, which implies that, once $P_{i}$ exceeded $\lambda_{2}$, response probability increased following $\mathrm{CR}$ trials and decreased following $\overline{\mathrm{CR}}$ trials. The smaller $\theta_{2}$ for the three Ss requiring two $\theta$ s indicates that probability decreased at a slower rate following a $\overline{\mathrm{CR}}$ trial than it increased following a CR trial.

The pattern in Group A was more complex. A single operator was sufficient for three Ss, while the remainder required two. Of the two-operator Ss, two exhibited a common $\lambda$, approached at different rates depending upon whether or not a CR occurred. In one case, for all practical purposes probability changed following only $\overline{\mathrm{CR}}$ trials. The remaining $\mathrm{Ss}$ all required two $\lambda \mathrm{s}$ and, as with Group C, $\lambda_{1}>\lambda_{2}$. The cats (Table 2) provided a pattern of parameters similar to that of the Group A dogs: Ss were scattered across the four assumption categories; $\lambda_{1}$ exceeded $\lambda_{2}$ and $\lambda_{1}$ was extremely high.

Table 1

Best-Fit Parameters for Individual Subjects in Groups A and C

\begin{tabular}{|c|c|c|c|c|c|c|}
\hline Group & $\mathbf{K}$ & $\theta_{1}$ & $\lambda_{1}$ & $\theta_{2}$ & $\lambda_{2}$ & $\begin{array}{l}\text { Assump- } \\
\text { tion* }\end{array}$ \\
\hline \multirow{14}{*}{$\mathrm{C}$} & 34 & .11427 & .81776 & .11427 & .08683 & $\mathrm{C}$ \\
\hline & 110 & .16448 & .69893 & .16448 & 20278 & C \\
\hline & 54 & .22156 & .93074 & .22156 & .15753 & $\mathrm{C}$ \\
\hline & 2 & .14564 & .78315 & .14564 & .03745 & $\mathrm{C}$ \\
\hline & 10 & .20504 & .80721 & .20504 & .06646 & $\mathrm{C}$ \\
\hline & 59 & .12142 & .80881 & .12142 & .02291 & $\mathrm{C}$ \\
\hline & 2 & .18911 & .97289 & 18911 & .11258 & $\mathrm{C}$ \\
\hline & 117 & .24591 & .79729 & .24591 & .17238 & $\mathrm{C}$ \\
\hline & 1 & .21112 & .98475 & 21112 & .04900 & C \\
\hline & 145 & .13609 & .76436 & .13609 & .07480 & $\mathrm{C}$ \\
\hline & 91 & .11226 & .72045 & .11226 & .07085 & $\mathrm{C}$ \\
\hline & 15 & .15993 & .87479 & .10790 & .03957 & D \\
\hline & 2 & .69106 & .39008 & .12623 & .02205 & D \\
\hline & 2 & .09887 & .87258 & .07125 & .02853 & $\mathrm{D}$ \\
\hline \multirow{14}{*}{ A } & 2 & .01458 & .99071 & .01458 & .99071 & A \\
\hline & 20 & .01075 & .97652 & .01075 & .97652 & A \\
\hline & 2 & .03288 & .98255 & .03288 & .98255 & A \\
\hline & 16 & .00874 & 1.00000 & .03009 & 1.00000 & B \\
\hline & 10 & .00000 & .90369 & .07766 & .90369 & B \\
\hline & 125 & .13237 & .94894 & .13237 & .20092 & $\mathrm{C}$ \\
\hline & 127 & .09499 & .99108 & .09499 & .19537 & $\mathrm{C}$ \\
\hline & 1 & .17427 & .97270 & .17427 & .04941 & C \\
\hline & 1 & .15880 & .94257 & .15880 & .09053 & $\mathrm{C}$ \\
\hline & 80 & .09270 & .99076 & .09270 & .07041 & C \\
\hline & 165 & .10869 & .97823 & .10869 & .03097 & $\mathrm{C}$ \\
\hline & 31 & .05758 & .98420 & .05758 & .02006 & $\mathrm{C}$ \\
\hline & 1 & .10514 & .88683 & .07572 & .01313 & D \\
\hline & 36 & .12312 & 98009 & .05931 & .04880 & $\mathrm{D}$ \\
\hline
\end{tabular}

*Note that when $S$ required a common $\theta$ or $\lambda$, the same value is entered under $\theta_{1}$ and $\theta_{2}$ or $\lambda_{1}$ and $\lambda_{2}$. The assumption categories are described in the text.
Table 2

Best-Fit Parameters for Individual Cat Subjects

\begin{tabular}{rrrrrr}
\multicolumn{1}{r}{$\mathrm{K}$} & \multicolumn{1}{c}{$\theta_{1}$} & \multicolumn{1}{c}{$\lambda_{1}$} & \multicolumn{1}{c}{$\theta_{2}$} & \multicolumn{1}{c}{$\lambda_{2}$} & $\begin{array}{c}\text { Assump- } \\
\text { tion }\end{array}$ \\
\hline 196 & .01046 & .91508 & .01046 & .91508 & $\mathrm{~A}$ \\
511 & .02162 & .43197 & .02162 & .43197 & $\mathrm{~A}$ \\
83 & .03307 & 1.00000 & .00045 & 1.00000 & $\mathrm{~B}$ \\
17 & .05508 & .94803 & .05508 & .04764 & $\mathrm{C}$ \\
64 & .17966 & .88717 & .17966 & .04232 & $\mathrm{C}$ \\
154 & .09927 & .96678 & .09927 & .01476 & $\mathrm{C}$ \\
28 & .20376 & .88979 & .20376 & .06660 & $\mathrm{C}$ \\
13 & .37463 & .66163 & .37463 & .16808 & $\mathrm{C}$ \\
227 & .05830 & 1.00000 & .05830 & .03756 & $\mathrm{C}$ \\
6 & .05837 & 1.00000 & .05837 & .02324 & $\mathrm{C}$ \\
20 & .70012 & .57071 & .70012 & .11015 & $\mathrm{C}$ \\
53 & .10273 & .88537 & .10273 & .05530 & $\mathrm{C}$ \\
2 & .05142 & 1.00000 & .05142 & .00546 & $\mathrm{C}$ \\
2 & .06979 & .96580 & .06979 & .02164 & $\mathrm{C}$ \\
44 & .08380 & 1.00000 & .08380 & .03328 & $\mathrm{C}$ \\
1 & .07438 & 1.00000 & .07438 & .02186 & $\mathrm{C}$ \\
7 & .07887 & 1.00000 & .07887 & .06569 & $\mathrm{C}$ \\
2 & .10795 & .82525 & .10795 & .07214 & $\mathrm{C}$ \\
1400 & .50329 & .77748 & .69783 & .24725 & $\mathrm{D}$ \\
\hline
\end{tabular}

*Note that when $S$ required a common $\theta$ or $\lambda$, the same value is entered under $\theta_{1}$ and $\theta_{2}$ or $\lambda_{1}$ and $\lambda_{2}$. The assumption categories are described in the text.

As reported by Wahlsten and Cole (1972), mean performance level for Group A exceeded that for Group C. This difference is reflected mainly in $\lambda_{1}$ : The Group A $\lambda_{1}$ was larger than the Group $\mathrm{C} \lambda_{1}$ in Assumption $C$ and D Ss $[t(10,8)=4.09]$. Group $C \theta$ was greater than that for Group A (Assumption A and C Ss) $[t(10,9)=3.57]$, indicating more trial-by-trial variability in response probability in the former group.

\section{DISCUSSION}

Of the theoretical positions described in the introduction, the present avoidance data of both dogs and cats are most consistent with that suggested by Hilgard and Marquis (1940) and by Bolles et al (1966). Phase 1 can be interpreted to be a phase during which $S$ identifies the environmental contingencies and, in some sense, selects a response. Upon entering Phase 2, the CR trial serves to increase the likelihood of the selected response. In more recent developments (Bolles, 1971), the selected response may be considered to be one of a limited set of possible species-specific defense reactions (SSDRs). On those trials in which a response occurs, the avoidance contingency itself is sufficient to increment and maintain response levels. The drop in response probability which is associated with $C R$ trials once $P_{i}$ exceeds $\lambda_{2}$, while not predicted by the theory, is not inconsistent with it. The minority of Ss exhibiting a single performance limit common to both $\mathrm{CR}$ and $\overline{\mathrm{CR}}$ trials also do not behave in a manner inconsistent with the theory, though their data do not constitute specific support for it.

If the function of the CS- $\overline{C R}$-UCS trial is merely to aid in response selection, thereby increasing its 
likelihood of occurrence, then the results with those dogs trained with the classical conditioning operations are also consistent with the Bolles theory. Presumably, the number of SSDRs is limited and functionally appropriate to the context. Thus, conditioned paw movement, which ordinarily would avoid aversive stimulation, is not only to be expected but also one of the few responses possible. When it is punished, there is some reduction in potential response level, thus accounting for a lower $\lambda_{1}$ in the classical preparation. The response, being one of the few SSDRs characte ristic of the species for such situations, may be attenuated on UCS trials, but not eliminated. The larger $\theta$ associated with the classical contingency does indicate wider variations in response probability throughout training, possibly reflecting the maladaptive effect of the UCS on CR trials.

In contrast, the present avoidance data are difficult to reconcile with two-factor theory. Given $\lambda_{1} \geqslant \lambda_{2}>P_{o}$, two of the outcomes are consistent with the theory: Response probability increases on both $\mathrm{CR}$ and $\overline{\mathrm{CR}}$ trials until $P_{i}>\lambda_{2}$. Thereafter the effect is, if anything, the reverse of what would be expected: For two-operator Ss $P_{i}$ decreases following $\overline{C R}$ trials and increases following CR trials. The two-factor theory requires that CS-CR-UCS trials maintain fear level, which means that its functional effect should be to increase response likelihood. While the theory does not requirc large drops in response probability following CR trials, certainly there is no basis for the increment obtained for both one- and two-operator $S$ s. Requiring as it does the acquisition of fear on $\overline{C R}$ trials, the two-factor theory also is in difficulty with the classically trained Ss, since in all $S s P_{i}$, once past $\lambda_{2}$, decreased following $\overline{C R}$ trials.

The two-phase model differs from the Bush-Mosteller model (Bush \& Mosteller, 1955) in two ways. First, on a formal level, a first phase of no change in response probability is assumed in the present model. That the best-fit parameters yielded $K \geqslant 1$ illustrates that the first phase is necessary. Phase 2 of the present model is formally identical to the Bush-Mosteller model. Second, in their application to avoidance behavior, Bush and Mostelle $r$ assumed that $\lambda_{1}=\lambda_{2}$, a simplifying assumption not made here. The large and consistent difference between $\lambda_{1}$ and $\lambda_{2}$ shows that this simplifying assumption cannot be made.

While Hoffman's model (Hoffman, 1965) makes formal predictions comparable to those expected based on past application of the Bush-Mosteller model, it cannot be reconciled quite as easily with the present data. This is because the Hoffman model is based upon specific assumptions concerning the acquisition of fear and its relationship to the acquisition of an avoidance response which do not permit a reconciliation. It would appear that modifications would be required in the theoretical mechanisms assumed by Hoffman in the development of his model.
In the present study the avoidance operations resulted in a higher level of performance than did the classical operations. This is not a general result. Such investigators as Gormezano and Coleman (1973), Kimble et al (1955), Logan (1951), and Moore and Gormezano (1961) have found that higher levels of performance are obtained with classical than with avoidance operations. These instances uniformly involved either the eyelid or the nictitating membrane reflex.

There are two perspectives from which to view the discrepancy between the leg flexion and membrane reflexes. As discussed by Gormezano (1965, pp. 48-50), the difference may be a result of counterconditioning an incompatible response. Girden (1938) and Sheffield (1948) drew specific attention to the possibility of counterconditioning by noting that, respectively, leg extension and termination of running frequently occurred to the UCS when conditioned flexion and running responses were in progress at the point of UCS application. Such counterconditioning does not seem likely with either the eyelid or nictitating membrane response, i.e., administering a UCS to the orbital region does not result in a reflex opening of a closed eye. If counterconditioning does account for the discrepancy across response systems, then an interpretation within the Bolles framework is less attractive unless it is additionally assumed that the set of SSDRs available to $S$ is contingent in part upon the response state of the organism at UCS occurrence.

Another perspective is that the difference between the leg flexion and membrane preparations reflects the evolutionary and behavioral history of those response systems. Leg movements do provide locomotion and are structured, therefore, entirely to avoid potentially aversive stimulation. Eyelid movements occur to stimuli when only a brief span of time separates warning from aversive stimuli and when, therefore, there is no response mechanism available entirely to avoid aversive stimuli. The response mitigates rather than avoids. It is reasonable to expect, therefore, that positive transfer would be higher to avoidance operations with leg flexion responses and to classical operations with membrane responses. This perspective is fully compatible with the Bolles theory, particularly when it is recognized that the mitigating, rather than avoidance, function of the membrane would make response punishment (UCS occurrence while the response is in progress) less salient in the transfer (laboratory) setting.

One final point merits note. The two-phase model has been successfully applied to classical conditioning performance in both rabbits and humans (Prokasy, 1973). In that research, with either the eyelid or nictitating membrane reflex, the large majority of data protocols could be described by a single operator (i.e., Assumption A). Those protocols which did require two operators yielded, as did this study, $\lambda_{1}>\lambda_{2}$. The large differences in species, response systems, training 
procedures, and number of trials preclude identifying the variables that control the proportion of one-operator Ss but, insofar as comparisons can be made, the pattern of parameter estimates obtained in this study does parallel that of the earlier research.

\section{REFERENCES}

Bolles, R. C. Species-specific defense reactions. In F. R. Brush (Ed.), Aversue conditioning and learning. New York: Academic Press, 1971. Pp. 183-233.

Bolles, R. C., Stokes, L. W., \& Younger, M. S. Does CS termination reinforce avoidance behavior? Journal of Comparative \& Physiological Psychology, 1966, 62, 201-207.

Bush, R. R., \& Mosteller, F. Stochastic models for learning. New York: Wiley, 1955.

Chandler, J. P. STEPIT: finds local minima of a smooth function of several parameters. (CPA 312) Behavior Science, 1969, 14, 81-82.

Girden, E. Conditioning and problem-solving behavior. American Journal of Psychology, 19 38, 51,677-686.

Gormezano, I. Yoked comparisons of classical and instrumental conditioning of the eyelid response; and an addendum on "voluntary responders." In W. F. Prokasy (Ed.), Classical conditioning. New York: Appletion-Century-Crofts, 1965. Pp. 48-70.

Gormezano, I., \& Coleman, S. R. The law of effect and CR contingent modification of the UCS. Conditional Reflex, $1973,8,41-56$.

Hilgard, E. R., \& Marquis, D. G. Conditioning and learning. New York: Appleton-Century-Crofts, 1940.

Hoffman, H. S. Theory construction through computer simulation. In W. F. Prokasy (Ed.), Classical conditioning: A symposium. New York: Appleton-Century-Crofts, 1965 Pp. 107-117.

Kimble, G. A., Mann, L. I., \& Dufort, R. H. Classical and instrumental eyelid conditioning. Journal of Experimenta Psychology, 1955, 49, 407-417.

Logan, F. A. A comparison of avoidance and nonavoidance eyelid conditioning. Journal of Experimental Psychology, $1951,42,391-393$.

Moore, J, W., \& Gormezano, I. Yoked comparisons of instrumental and classical ey elid conditioning. Journal of Experimental Psychology, 1961,62, 552-559.

Nielson, H. C., \& Davis, K. B. Effect of frontal ablation upon conditioned responses. Journal of Comparative \& Physiological Psychology, 1966, 61, 380-387.

Norman, M. F. A two-phase model and an application to verbal discrimination learning. In R. C. Atkinson (Ed.), Studies in mathematical psychology. Stanford: Stanford University Press, 1964.

Prokasy, W. F. Developments with the two-phase model applied to human ey elid conditioning. In A. H. Black and W. F.
Prokasy (Eds.), Clossical conditioning $I I$. New York: Appleton-Century-Crofts, 1972. Pp. 119-147.

Prokasy, W. F. A two-phase model account of aversive classical conditioning performance in humans and rabbits. Learning \& Motivation, 1973, 4, 247-258.

Prokasy, W. F., Williams, W. C., Lee, W. Y. M., \& Kumpfer, K. L. Two-phase model analysis of the effects of interstimulus interval and masking task in human aversive conditioning. Memory \& Cognition, 1974, 2, 206-210.

Sheffield, F. D. Avoidance training and the contiguity principle. Journal of Comparative \& Physiological Psychology, 1948, 41 165-177.

Theios, J. Finite integer models for learning in individual subjects. Psychological Review, 1968, 75, 292-307.

Theios, J. Mathematical models for aversive conditioning. In $F$ R. Brush (Ed.), Aversive conditioning and learning. New York: Academic Press, 1971. Pp. 297-346.

Wahlsten, D. C., \& Cole, M. Classical and avoidance training of leg flexion in the $\operatorname{dog}$. In A. H. Black and W. F. Prokasy (Eds.), Classical conditioning II. New York: Appleton-Century-Crofts, 1972. Pp, 379-408.

\section{NOTES}

1. I would like to thank J. Michael Cassady and Michael Colp for their efforts in providing the dog protocols and Fiatit Nielson for his efforts in providing the cat protocols.

2. The maximum likeliho od estimates obtained with STEFIT are not entirely independent of the start values. For example, with 10 different sets of start values, each member of a set selected from a table of random numbers, it is not uncommon for the maximum likelihood obtained with up to three of the sets to be substantially lower than that of the rest. The likelihoods obtained with the remaining sets are typically close in value and yield similar parameter estimates. The procedure adopted was to obtain the highest maximum likelihood estimate provided by STEPIT from three ind ependent sets of start points. This means that an occasional protocol will reflect a local, rather than global, maximum likelihood. In spite of this, tests of goodness of fit between Monte Carlo generated expectations and obtained statistics suggest that the model fits reasonably well Employing such statistics as mean response probability over blocks of 10 trials, approximately $10 \%$ of the obtained values fall outside the $95 \%$ confidence interval. These departures, though greater than the number expected, reveal no obvious pattern, thus suggesting idiosyncracies rather than any systematic departure of data from model.

(Received for publication November 9, 1973; revision accepted May 7,1974 .) 